J. Phys. IV France 138 (2006) 265-274

(C) EDP Sciences, Les Ulis

DOI: $10.1051 / \mathrm{jp} 4: 2006138031$

\title{
Le pôle de métrologie de SOLEIL
}

\author{
M. Idir ${ }^{1, *}$, S. Brochet ${ }^{1}$, A. Delmotte ${ }^{1}$, B. Lagarde $^{1}$, P. Mercere ${ }^{1}$, T. Moreno ${ }^{1}$, \\ F. Polack ${ }^{1}$ et M. Thomasset ${ }^{1}$ \\ ${ }^{1}$ Synchrotron SOLEIL - Division Expériences, France
}

Résumé. Le Pôle de METROLOGIE de SOLEIL a pour objet de créer sur le synchrotron SOLEIL, une plateforme constituée :

- 'une ligne de lumière utilisant le rayonnement synchrotron (métrologie dite à la longueur d'onde)

- d'un laboratoire de métrologie associé (métrologie dite « classique »)

Ces deux types de Métrologie sont l'une et l'autre indispensables pour soutenir l'activité de recherche instrumentale en optique $\mathrm{X}$ et X-UV. Ce projet de pôle de METROLOGIE ne répondra pas seulement aux besoins des groupes chargés de l'équipement du synchrotron SOLEIL en optiques et détecteurs mais aussi pour préparer, tester et mettre au point les postes expérimentaux, ce qui concerne déjà une large communauté d'utilisateurs. Il sera aussi largement ouvert, dès sa mise en service, à l'ensemble de la communauté scientifique concernée par l'instrumentation X et XUV en Ile de France, en France, voire même en Europe si la demande continue de croître plus vite que l'offre dans ce domaine.

Ligne de lumière - Métrologie à la longueur d'onde

La ligne de lumière sera équipée de plusieurs stations permettant de mesurer, dans la plus grande partie du spectre couvert par le synchrotron, les paramètres photométriques qui caractérisent les éléments optiques, tels que : la réflectivité de surfaces, l'efficacité de diffraction des réseaux, la diffusion des surfaces ou l'efficacité des détecteurs X et X-UV et la calibration absolue. Cette installation pourra servir également à développer des instruments et des diagnostics nécessaires à la caractérisation des faisceaux de rayons $\mathrm{X}$ (intensité, taille, degré de cohérence, polarisation etc.)

Métrologie Classique

La métrologie des surfaces optiques est devenue une nécessité critique pour les laboratoires et les industries qui utilisent les photons X et X-UV (synchrotrons, centres laser, etc. .). En effet, les progrès de calcul et de conception des systèmes optiques pour ces longueurs d'onde (optiques de microfocalisation, monochromateurs, diagnostics d'imagerie) font que les performances de ces instruments sont désormais limitées par les imperfections de fabrication des composants optiques. La métrologie des surfaces optiques est donc une nécessité impérieuse pour tous les acteurs du domaine, qui se doivent d'effectuer les contrôles appropriés. Cette pression s'exerce aussi sur les moyens utilisés pour effectuer ces mesures, car les incertitudes de mesure actuelles, notamment en ce qui concerne la régularité des surfaces, sont loin d'être négligeables vis à vis des tolérances demandées. Il est donc indispensable de faire évoluer les instruments de mesure et d'obtenir des gains significatifs de précision. Un travail particulier est en cours au laboratoire de Métrologie pour développer à côté des instruments commerciaux, des instruments prototypes sur des concepts originaux (mesures de profils de surface et mesures d'angle). Dans cet article, nous donnons des détails des choix techniques utilisés sur la ligne de METROLOGIE et TESTS et des performances attendues et nous décrirons le laboratoire de METROLOGIE en donnant des exemples d'optiques récemment testées.

\section{INTRODUCTION}

Avec la construction de nombreux centres de rayonnement synchrotron (RS) dans le monde, l'intérêt et l'activité de la gamme des énergies des X "mous" et de l'extrême ultraviolet (gamme X-UV) a connu un développement considérable depuis quelques années. L'intérêt de cette région spectrale ne s'est pas uniquement focalisé sur les applications scientifiques et techniques du RS ; il y a eu en parallèle une croissance similaire dans les domaines de l'astronomie $\mathrm{X}$, la physique atomique, la fluorescence $\mathrm{X}$, les lasers X et pour la lithographie extrême UV. En revanche, actuellement en France, la métrologie

*Mourad.idir@synchrotron-soleil.fr 
associée à ces gammes d'énergie n'a pas connu d'expansion similaire et les besoins des utilisateurs sont généralement peu ou pas satisfaits.

Avec la réalisation du synchrotron SOLEIL, l'instrumentation française (au sens large) utilisatrice de photons X et XUV bénéficie d'une opportunité exceptionnelle pour développer ses études dans ces domaines tournés vers les développements du futur. Une large communauté de scientifiques a manifesté son intérêt pour pouvoir bénéficier de moyens métrologiques dès le début du fonctionnement du synchrotron ${ }^{1}$.

Un projet de ligne de métrologie ${ }^{2}$ a donc été conçu par un groupe constitué d'utilisateurs et de métrologues, puis présenté au Conseil Scientifique de SOLEIL et approuvé par celui-ci. Le projet actuel prévoit de créer sur le synchrotron SOLEIL, un pôle de métrologie constitué :

- de lignes de lumière utilisant le rayonnement synchrotron (métrologie dite à la longueur d'onde) en collaboration avec le Laboratoire National d'Essais (LNE) et le CEA DIF

Les lignes de lumière permettent par exemple de mesurer la réflectivité de surfaces optiques, l'efficacité de diffraction des réseaux, la diffusion des surfaces et l'efficacité des détecteurs X et XUV et la calibration absolue. Avec ce dispositif, le Laboratoire National d'Essais (LNE) se dotera d'un outil d'étalonnage commun à la gamme des photons UV et des photons X dont l'exploitation est confiée à 3 laboratoires du LNE. Un intérêt majeur de ce projet est la constitution d'une source primaire de rayonnement X-UV : la ligne de métrologie pourra fonctionner comme source étalon, avec un flux spectral calculable et devenir ainsi la référence nationale pour ces rayonnements photoniques. Pour le CEA DIF, l'utilisation de la ligne de métrologie est très importante pour la caractérisation de tous les diagnostics de la LIL et du futur laser Mégajoule

- d'un laboratoire de métrologie associé (métrologie dite « classique »)

Le laboratoire de Métrologie utilise des instruments soit commerciaux soit en cours de développement (prototype) pour les mesures des composants optiques (miroirs, cristaux, réseaux, etc. .).

Le présent document fait état de ce projet en présentant le contexte du synchrotron, les différentes communautés intéressées par la ligne et le laboratoire de métrologie.

\section{LA LIGNE DE MÉTROLOGIE ET TESTS}

Dans le projet actuel, la ligne de Métrologie et Tests est composée de trois branches de rayonnement utilisables simultanément en exploitant $8 \mathrm{mrad}$ de divergence horizontale de l'aimant de courbure D05-1.

La branche dite «VUV 10-100 eV » est obtenue en déviant horizontalement une partie (entre 1 et 2 mrad) du faisceau émis par l'aimant de courbure D05-1 à l'aide d'un miroir sphérique placé en tête de ligne travaillant à un angle de $11^{\circ}$. La branche dite « X mous $30-2000 \mathrm{eV}$ » est obtenue en déviant horizontalement une partie ( $2 \mathrm{mrad})$ du faisceau émis par l'aimant de courbure D05-1 à l'aide d'un miroir sphérique placé en tête de ligne travaillant à un angle de $2^{\circ}$. La partie du faisceau non déviée forme la partie dite « X durs $100-28000 \mathrm{eV} »$. La figure 1 représente l'implantation au sol de la ligne de METROLOGIE et TESTS avec les trois branches.

\subsection{Branche VUV}

La branche VUV de la ligne Métrologie doit pouvoir travailler dans la gamme spectrale comprise entre quelques eV eV et $100 \mathrm{eV}$. Le principe optique retenu est un monochromateur de type TGM300 de Jobin Yvon [4]. Pour couvrir le domaine spectral, le monochromateur est composé de deux réseaux toriques de 550 t/mm utilisés à l'ordre -1 (rasant en sortie) entouré de deux miroir toriques $\mathrm{M}_{1}$ et $\mathrm{M}_{3}$ fonctionnent

\footnotetext{
${ }^{1}$ Workshop "métrologie" du 30 mai 2001.

2"The Metrology beam line at SOLEIL" par M. Idir, J. Underwood, F. Polack, M.C. Lépy et P. Stemmler.
} 


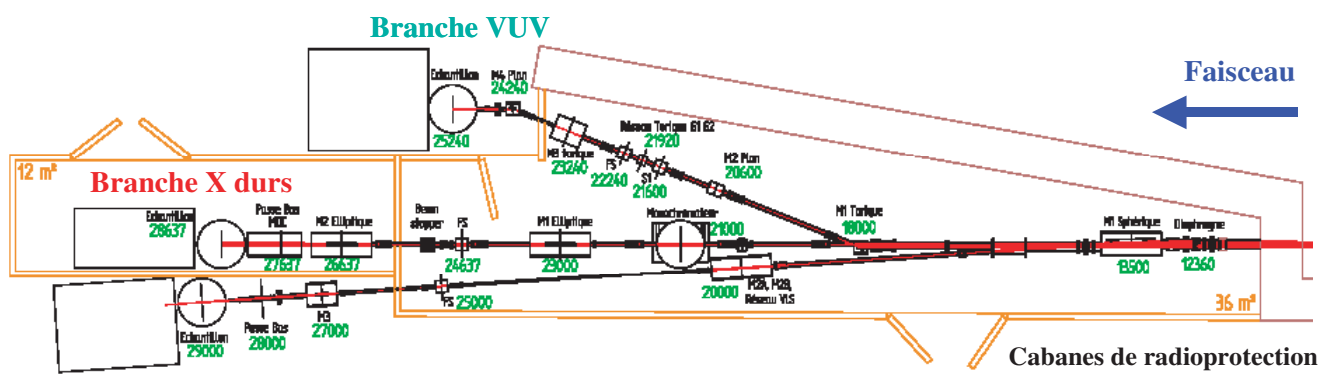

Branche XUV

Figure 1. Implantation au sol de la ligne de METROLOGIE et TESTS avec les trois branches.

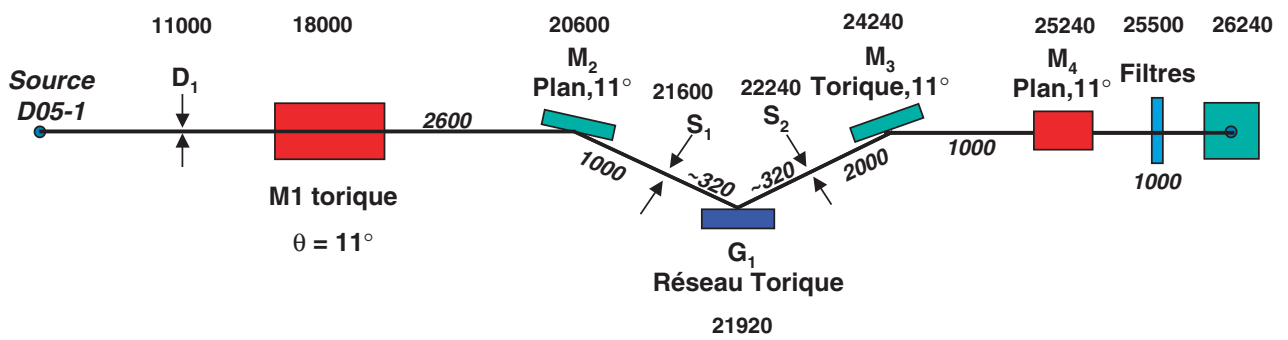

Figure 2. Schéma de principe de la branche VUV de la ligne de METROLOGIE et TESTS.

à $11^{\circ}$ ainsi que de miroirs plans (M2 et M4). La figure 2 montre le schéma de principe de la branche VUV de la ligne de METROLOGIE et TESTS

A titre dexemple de performances, pour une énergie de $\mathrm{E}=15 \mathrm{eV}$, la taille du faisceau focalisé est de $210 \mu \mathrm{m} \times 950 \mu \mathrm{m}$ FWHM (en considérant $1.5 \mu \mathrm{rad} \mathrm{rms}$ d'erreur de pente sur les optiques), la résolution spectrale est d'environ 300 et le flux de $\sim 6 \times 10^{11}$ photons/s avec une pureté spectrale meilleure que $10^{-4}$.

\subsection{Branche XUV}

Cette branche travaille dans la gamme spectrale 30 - 2000 eV. La résolution spectrale est obtenue grâce à un monochromateur du type Hettrick - Underwood utilisant trois réseaux VLS plans. Pour couvrir le domaine spectral, le monochromateur est composé d'un miroir sphérique (M2-XUV) et de trois réseaux VLS plans de respectivement 75,300 et $1200 \mathrm{t} / \mathrm{mm}$ utilisés à l'ordre -1 (rasant en sortie) et avec un angle de déviation de $175^{\circ}$.

Le miroir M3-XUV à $1.25^{\circ}$ (miroir plan courbable) focalise verticalement le faisceau transmit par $\mathrm{S}_{2}$ sur le plan image situé à $31.0 \mathrm{~m}$ de la source. Le premier miroir recevant le faisceau M1XUV permet de focaliser le faisceau dans la direction horizontale au niveau de l'échantillon $(31.0 \mathrm{~m}$ de la source).Finalement, le coupeur d'ordre permet d'éliminer les harmoniques d'ordre supérieur du monochromateur. Il est constitué d'un barillet de filtres en transmission (F) et d'un filtre passe bas en énergie (miroirs plans $\mathrm{MPB}_{1}, \mathrm{MPB}_{2}$ et $\mathrm{MPB}_{3}$ ) ce qui permet d'avoir un taux de réjection d'harmoniques $>10^{-3}$ sur l'ensemble du domaine spectral. Le principe de cette branche est décrit sur la figure 3 .

A titre d'exemple de performances, le faisceau arrivant sur l'échantillon est montré sur la figure 4 pour une énergie de $\mathrm{E}=92.52 \mathrm{eV}(\lambda=13.4 \mathrm{~nm})$ (simulation effectué par tracé de rayons Monte Carlo). La taille du faisceau focalisé est de $300 \times 50 \mu \mathrm{m}$ FWHM (en considérant $1.5 \mu \mathrm{rad}$ rms d'erreur de pente sur les optiques), la résolution spectrale est de environ 2500 et le flux de $\sim 3.5 \times 10^{10}$ photons/s avec une pureté spectrale meilleure que $10^{-3}$. 


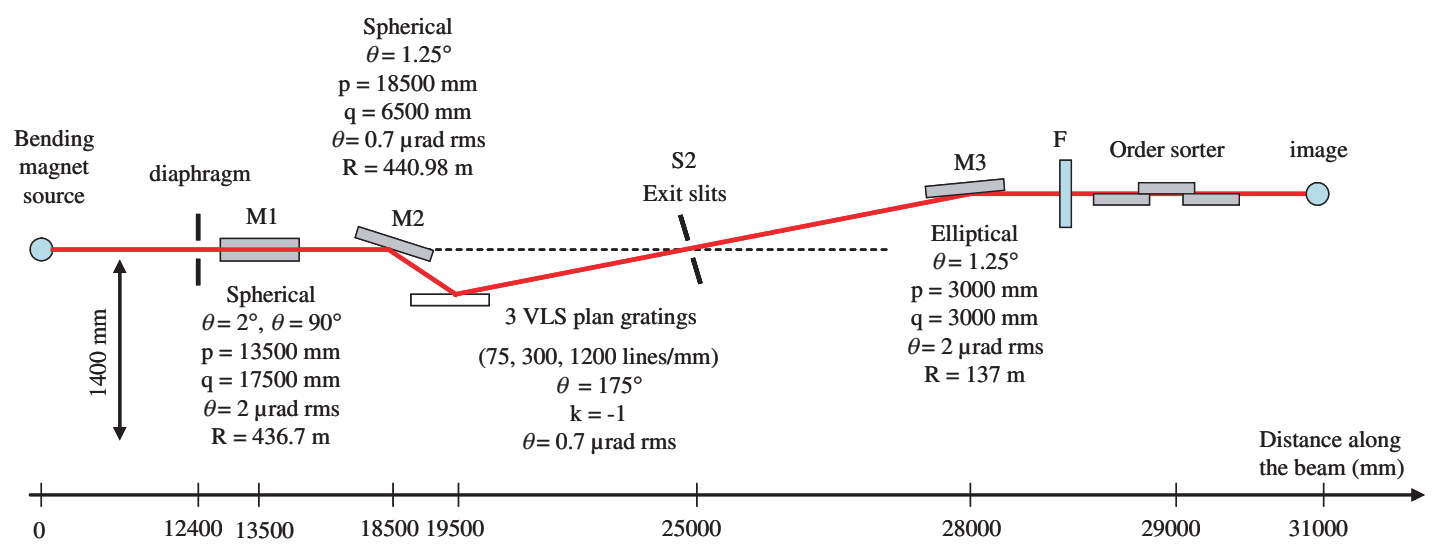

Figure 3. Schéma de principe de la branche XUV.

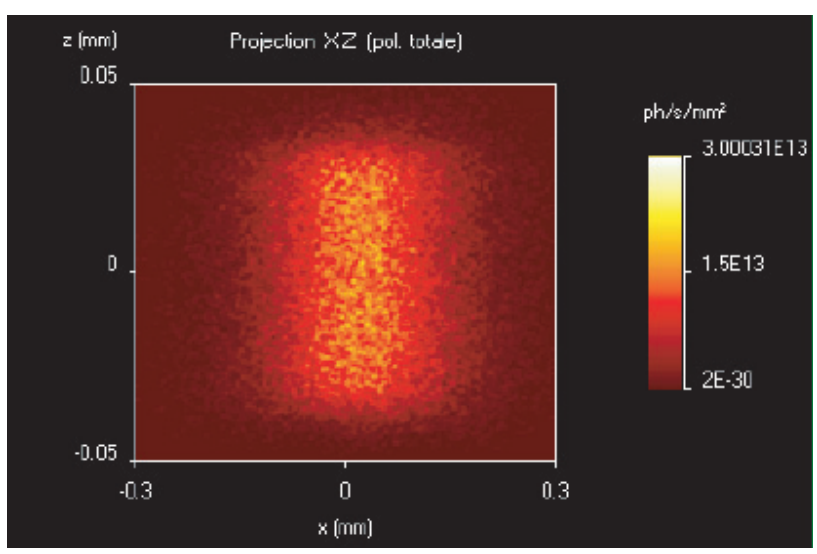

Figure 4. Faisceau XUV au niveau de l'échantillon pour $\mathrm{E}=92.5 \mathrm{eV}$.

\subsection{Branche X-durs}

La branche X durs de la ligne Métrologie doit pouvoir travailler dans la gamme d'énergie comprise entre 0.1 et $12 \mathrm{keV}$. Le principe optique retenue se décompose de la manière suivante :

Le premier système optique est un monochromateur à deux cristaux (MDC) équipé de Cristaux de Si 111 situé à $21 \mathrm{~m}$ de la source. A partir de la mécanique d'un monochromateur à deux cristaux, il est possible d'utiliser un monochromateur à réseaux multicouches (MRM) pour atteindre les basses énergies. Ce monochromateur est suivi d'un miroir elliptique ( $23 \mathrm{~m}$ de la source) qui permet de focaliser dans le plan vertical sur une fente de sortie située à $24.64 \mathrm{~m}$ de la source. La fente FC située à $12 \mathrm{~m}$ de la source permet de limiter la divergence du faisceau dans le plan vertical afin d'augmenter si nécessaire la résolution spectrale. Le miroir M2 situé à $2 \mathrm{~m}$ de la fente permet la focalisation du faisceau dans le plan vertical. Il est constitué d'un miroir plan sur courbeur pour permettre de faire varier la focalisation dans le plan vertical. Le faisceau peut donc être focalisé dans le plan vertical : sur l'échantillon (au centre du réflectomètre à $28.64 \mathrm{~m}$ de la source) ou à l'infini pour les études de diffusion par exemple. Trois modes de fonctionnement peuvent être utilisé sur cette branche : accès au faisceau blanc sans optique, accès au faisceau monochromatique focalisé et au faisceau monochromatique focalisé. 


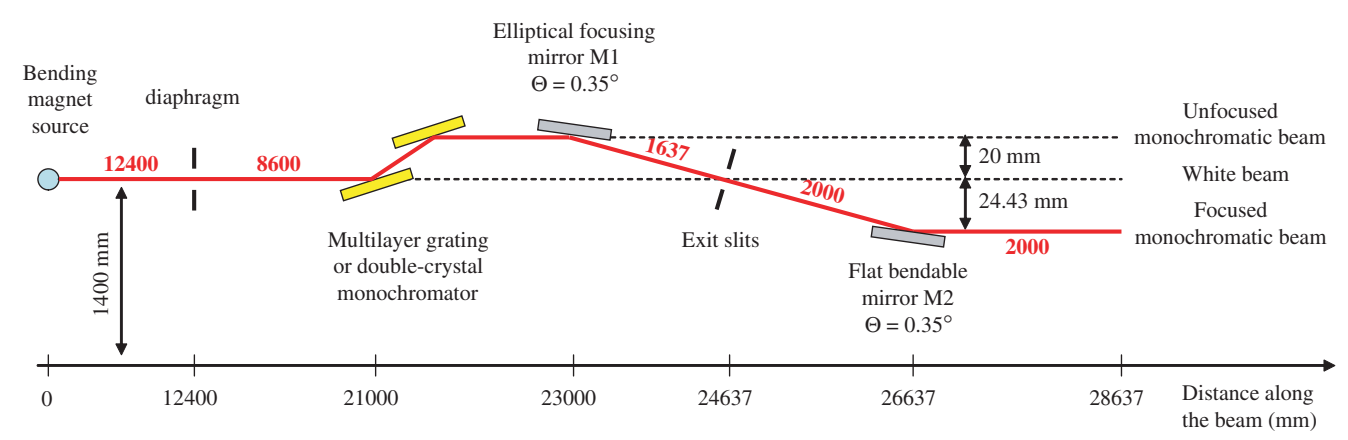

Figure 5. Schéma de principe de la branche X-durs.

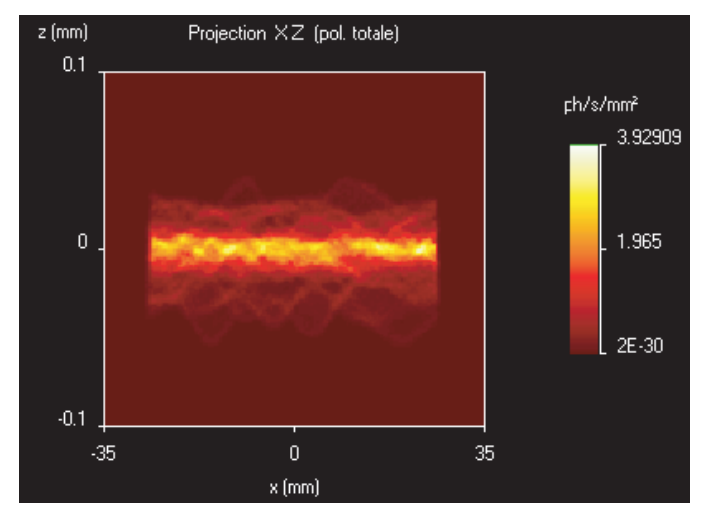

Figure 6. Faisceau X durs focalisé au niveau de l'échantillon (voir texte pour les détails).

A titre d'exemple de performances, le faisceau arrivant sur l'échantillon est montré sur la figure 4 pour une énergie de $\mathrm{E}=10 \mathrm{keV}(\lambda=0.124 \mathrm{~nm})($ simulation effectué par tracé de rayons Monte Carlo). La taille du faisceau focalisé est de $54 \mathrm{~mm} \times 60 \mu \mathrm{m}$ FWHM (en considérant $1.5 \mu \mathrm{rad}$ rms d'erreur de pente sur les optiques), la résolution spectrale est de environ 2500 et le flux de $\sim 10^{12}$ photons/s avec une pureté spectrale meilleure que $10^{-7}$.

La détermination absolue du flux de photons émis par un Aimant de courbure, avec une faible incertitude, pour en faire une source étalon primaire est une activité qui n'est actuellement développée qu'au PTB et au NIST. Pour ce genre de mesure, il peut être nécessaire d'avoir accès à du temps de faisceau spécifique, utilisable uniquement pour les besoins de la métrologie. En dépit de ces contraintes, cette activité est assurée à BESSY II pour les besoins du PTB ; ainsi les capacités de cette installation attirent les laboratoires du monde entier et en particulier de nombreux laboratoires d'astrophysique spatiale [5-6-7-8].

Le rayonnement synchrotron émis par les anneaux de stockage au niveau des aimants constitue une source étalon primaire puisque le flux photonique spectral $\left(\Phi_{\mathrm{E}}\right)$ émis est calculable à partir des relations électrodynamiques fondamentales (théorie de Schwinger) [9]. Ce calcul nécessite la connaissance de paramètres physiques tels que le champ magnétique, le courant et l'énergie des électrons et la géométrie de la mesure. La précision du calcul dépend évidemment de l'incertitude affectant ces différents paramètres

$$
\Phi_{\mathrm{E}}=\mathrm{f}(\mathrm{W}, \mathrm{B}, \mathrm{I}, \text { Géo })
$$

A priori, les paramètres géométriques ne posent pas de problème technique spécifique. En revanche, il faut pouvoir mesurer B, l'induction magnétique de l'aimant de courbure utiliser pour cette 

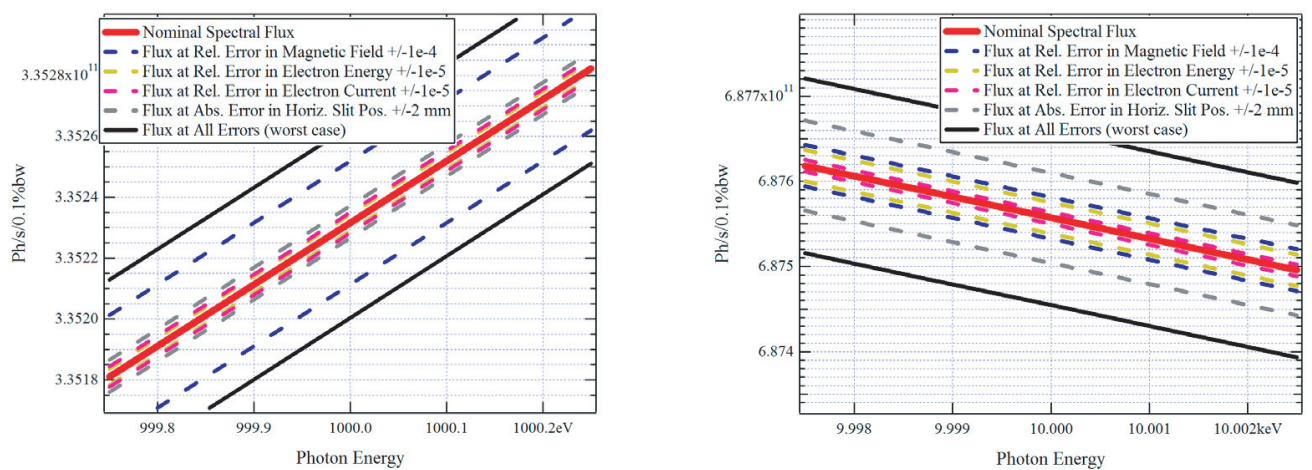

Figure 7. Calcul théorique du flux absolu pour $(\mathrm{E} \approx 1 \mathrm{keV}, 10 \mathrm{keV})$ en fonction des différents paramètres de la machine.

caractérisation. A BESSY, cette détermination s'effectue par sonde à résonance magnétique nucléaire (Incertitude typique à BESSY : $2 \cdot 10^{-4} \mathrm{~T}$ pour $\mathrm{B}=1,6 \mathrm{~T}$ ). Différentes méthodes devront être utilisées pour la mesure du courant d'électrons, I, selon l'intensité (et le nombre d'électrons) concernée. Enfin, l'énergie des électrons, $\mathrm{W}$, n'est pas aisée à déterminer simplement et nécessite de développer un équipement spécifique. A BESSY, deux méthodes sont utilisées : la rétrodiffusion Compton et la dépolarisation résonante de spin ; la comparaison des résultats obtenus par ces deux méthodes montre un accord avec les incertitudes relatives combinées de $2 \cdot 10^{-4}$. Une expérience de dépolarisation est déjà prévue par le groupe «machine» de SOLEIL et il suffit donc d'étudier la façon d'accorder les objectifs «machine » et « métrologie».

Les figures ci-après présentent les incertitudes théoriques accessibles à SOLEIL en fonction des différents paramètres de l'équation 1. Les incertitudes sont dominées par les facteurs géométriques. Pour SOLEIL, une incertitude relative de $3 \times 10^{-5}$ pour $\mathrm{E}=1 \mathrm{keV}$ et de $2 \times 10^{-4}$ pour $\mathrm{E}=10 \mathrm{keV}$ semble accessible (figure 7).

\section{LE LABORATOIRE DE MÉTROLOGIE OPTIQUE (LMO)}

L'histoire du laboratoire de métrologie optique de SOLEIL remonte à l'époque de LURE, l'ancien anneau de stockage situé sur le campus d'Orsay. Pour les besoins liés à la jouvence des lignes et aux futurs besoins en optique des lignes de lumière de SOLEIL, le groupe optique de LURE avait commencé à développer une instrumentation pour la métrologie des optiques XUV.

Les besoins croissants en terme de résolution spatiale et spectrale des utilisateurs des lignes de lumière synchrotron obligent les fabricants d'optiques à repousser toujours plus loin les possibilités de production en terme d'erreur de pente, de rugosité et de courbure pour des miroirs dont les dimensions peuvent atteindre $1 \mathrm{~m} 50$ de long. Les designers de lignes se doivent de demander aux fabricants des spécifications optiques en accord avec les besoins de la ligne mais aussi, être capables, en parallèle, de pouvoir qualifier les pièces optiques livrées par le fabricant.

Le rôle du laboratoire de métrologie de SOLEIL est donc, dans un premier temps, de se doter des instruments nécessaires à la qualification de ces objets. Dans un deuxième temps, de mesurer les pièces optiques livrées par le fabricant.

Les optiques synchrotrons pour le rayonnement XUV sont des optiques spécifiques : les courtes longueurs d'ondes empêchent l'utilisation de la réfraction. Les optiques fonctionnent par réflexion et par diffraction, tels les miroirs et les réseaux. Les angles d'incidence sont très rasants ce qui rend les optiques dissymétriques. Dans le cas particulier des miroirs toriques, elles sont allongées dans la direction d'incidence et étroite dans l'autre direction. Ceci peut conduire à une grande différence de 
rayon de courbure selon les 2 axes de l'optique ce qui rend la réalisation difficile et donc une qualité de surface encore plus complexe à atteindre.

On qualifie les défauts selon leur périodicité. On parle de la fréquence spatiale qui est l'inverse de la périodicité. Les défauts de fréquence spatiale élevée diffractent la lumière dans un angle proportionnel à la fréquence spatiale. Si cet angle est plus grand que la tache image de la source, on observe un halo de diffusion autour de l'image. Ce sont les défauts dits de rugosité. Les instruments qui mesurent de tels défauts sont les rugosimètres (ou microscopes interférentiels à balayage de phase) dont la résolution est de l'ordre de $0,1 \mathrm{~nm}$ et peuvent mesurer des défauts jusqu'à une fréquence spatiale de $100000 \mathrm{~mm}^{-1}$. Pour des fréquences plus élevées encore, on utilise un Microscope à Force Atomique (AFM).

L'AFM sert également à mesurer les formes des créneaux des réseaux de diffraction utilisés dans les monochromateurs des lignes dites «X-mous ». La connaissance de la forme réelle des créneaux ainsi que de leur période est très importante pour la caractérisation de leurs capacités de résolution spectrale. Les rugosimètres et les interféromètres sont des instruments disponibles commercialement ou dans des laboratoires proches et dont les précisions sont assez bonnes.

Cependant, la caractérisation des réseaux à l'aide d'un AFM est loin d'être triviale. La calibration des axes des piezo électriques de l'instrument est primordiale et n'est pas toujours bien maîtrisé par les constructeurs d'AFM.

Les défauts de basses fréquences sont appelés défauts de forme. Ces défauts peuvent apparaitre comme une succession de facettes qui défléchissent la lumière proportionnellement à l'erreur de pente locale. Lorsque la longueur d'onde est faible, cet effet prédomine sur la diffraction. La largeur de l'image d'une source ponctuelle est alors proportionnelle à l'erreur quadratique moyenne de pente de la surface éclairée. Pour les longueurs d'onde des lignes de lumière synchrotron XUV et pour les angles d'incidence les plus usuels, les fréquences qui se comportent ainsi sont celles inférieures à $0,3 \mathrm{~mm}^{-1}$. Les erreurs de pente à mesurer sont de l'ordre du $\mu$ rad. Les meilleures surfaces ont actuellement un défaut de forme meilleur que $1 \mu \mathrm{rad}$. La limite de précision des instruments de mesure doit être sensiblement inférieure à cette valeur.

C'est le cas des interféromètres qui mesurent l'écart entre la surface à mesurer et une surface de référence. L'erreur de pente peut être obtenue par dérivation de l'interférogramme mais cela exige une précision très élevée et une numérisation à un pas de l'ordre de $1 \mathrm{~mm}$. Un interféromètre commercial peut atteindre une précision de $\lambda / 500$ soit $1 \mu \mathrm{rad}$ de pente. De plus, un interféromètre effectue une mesure de comparaison avec une surface de référence. On ne peut donc mesurer que des surfaces proches de la surface de référence. Pour les miroirs courbes, il faut que la surface de référence ait un rayon de courbure proche de la surface à tester. Il faudra donc disposer d'un grand panel de surfaces de référence. De plus, le champ observable par un interféromètre est de l'ordre de $200 \mathrm{~mm}$ au maximum ce qui est bien inférieur aux dimensions de certaines optiques synchrotrons. Par contre, un interféromètre mesure un profil de pente à 2 dimensions. C'est donc pour cela que cet instrument est incontournable et permet des mesures « rapides » pour le montage des pièces optiques dans leur support mécanique.

Les rugosimètres et les interféromètres sont des instruments incontournables mais disponibles commercialement et dont les précisions sont assez bonnes. Le groupe Optique de SOLEIL a donc choisi de développer, pour commencer, un outil de mesure dont les caractéristiques requises n'existent pas dans le commerce.

\subsection{Le profilomètre optique : LTP (Long Trace Profiler)}

La plupart des profilomètres des laboratoires synchrotrons sont une version commerciale profondément modifiée par chaque laboratoire en fonction de ses besoins. Nous avons choisi de complètement développer cet instrument en interne. Une translation possédant un défaut de roulis-tanguage-lacet à mieux que $5 \mu \mathrm{rad}$ r.m.s. a été spécialement conçue pour l'instrument. L'interféromètre de Michelson, couramment utilisé sur les autres systémes LTP, a été remplacé par un interféromètre à polarisation assurant une excellente qualité de stabilité dans le temps. Nous avons atteint avec cet instrument une 


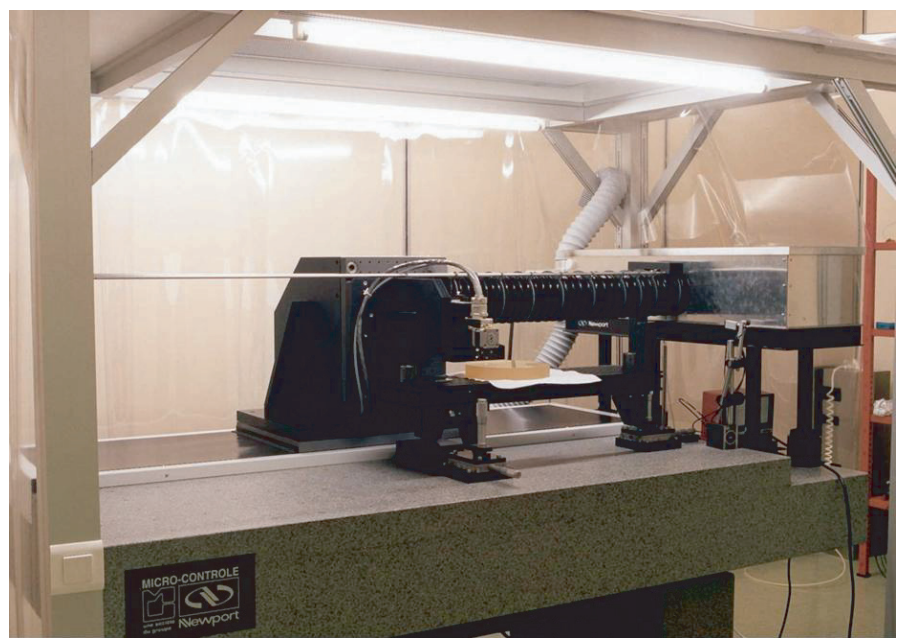

Figure 8. Le LTP installé en salle blanche ISO 7.

résolution de $0,3 \mu$ rad ce qui est meilleur que la version commerciale. Cette résolution a été déterminée par l'aide de mesures croisées avec un interféromètre à balayage de phase développé à l'IOTA et avec les profilomètres de ELETTRA, ESRF, BESSY, SLS, SPRING 8 [10-11].

Cet instrument est maintenant capable de mesurer des rayons de courbure compris entre $3 \mathrm{~m}$ et l'infini avec une précision de $0,1 \%$ pour des miroir de $1 \mathrm{~m}$ de long $^{2}$. Nous avons également étendu la mesure de pente locale de miroir à la mesure de densité trait locale de réseaux pour la métrologie des réseaux VLS.

Sur la tête optique du LTP ont été ajouté un interféromètre possédant une pupille de $50 \mathrm{~mm}$ ainsi qu'un analyseur de front d'onde de type Shack-Hartman développé en collaboration avec la société Imagine Optic à Orsay [12]. Cet instrument nous permet de mesurer avec une précision de $\lambda / 1000$ des optiques pour des rayons de courbure allant de $500 \mathrm{~mm}$ à l'infini.

\subsection{Le Microscope à Force Atomique (AFM)}

L'étude de la densité locale de traits des réseaux n'est pas suffisante pour qualifier un réseau utilisé dans les monochromateurs des lignes XUV. La forme de gravure du trait ainsi que le rapport cyclique (rapport entre la taille du trait gravé et la période du réseau) sont des paramètres importants qu'il est nécessaire de qualifier pour optimiser l'utilisation de l'objet. L'AFM est l'instrument qui caractérise, pour l'instant, le mieux ces paramètres.

Sa résolution est de $0,1 \mathrm{~nm}$ en hauteur.

Des versions commerciales de cet instrument sont capables de répondre à nos besoins. Avec peu de modifications mécaniques (surélévation du scanner de $60 \mathrm{~mm}$ pour permettre la mesure d'échantillon dont l'épaisseur peut varier de quelques $\mathrm{mm}$ à $60 \mathrm{~mm}$ ), nous utilisons un AFM répondant parfaitement à nos besoins.

\subsection{Le rugosimètre : ou microscope à balayage de phase}

De la même manière que pour l'AFM, une version commerciale de cet instrument est utilisée. Nous avons choisi de monter cet instrument sur une structure en forme de pont pour mesurer facilement des miroirs dont la longueur peut atteindre $1.50 \mathrm{~m}$. Divers objectifs interférentiels sont montés sur un 


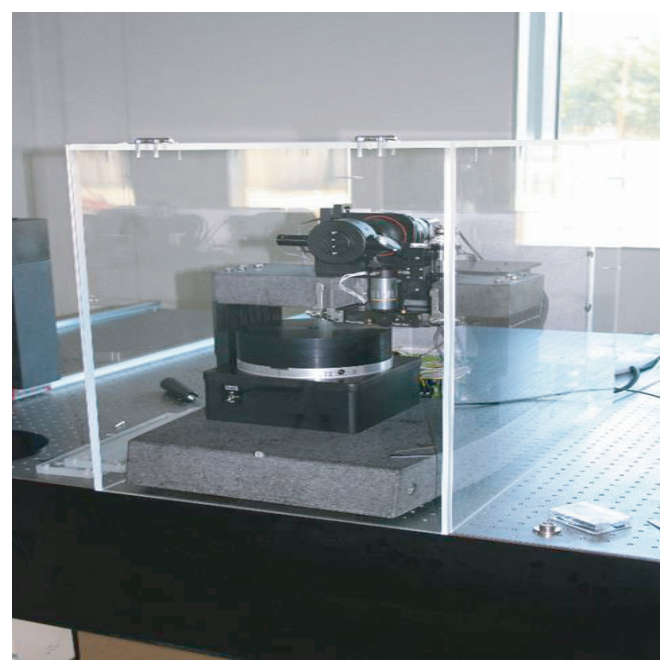

Figure 9. L'AFM installé en salle blanche et protégé des perturbations acoustiques par une boîte en plexiglas. Modèle : Nano-I de chez Pacific Nanotechnology.

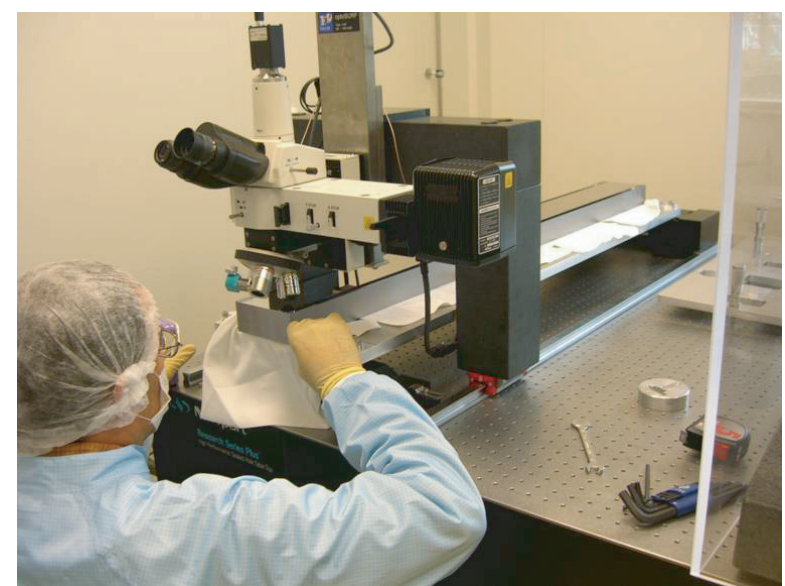

Figure 10. Le rugosimère. Modèle : Optosurf de chez EOTECH.

plateaux tournant nous permettant d'obtenir des mesures à diverses fréquences d'échantillonnage pour une résolution de $0,1 \mathrm{~nm}$ en hauteur.

L'AFM et le rugosimètre sont des instruments complémentaires pour l'étude des rugosités de miroirs puisqu'ils permettent de reconstituer expérimentalement la densité spectrale de puissance (PSD) de l'optique. Le LTP et l'interféromètre permettent également d'accéder aux fréquences les plus grandes. En optique pour le rayonnement XUV, ce sont les défauts de basses fréquences qui altèrent la résolution spatiale de l'optique.

Le laboratoire de métrologie est installé dans le bâtiment du synchrotron depuis juillet 2005 et a quasiment terminé la caractérisation des optiques des lignes de lumière de la phase I. Une cinquantaine de miroirs ont été caractérisés en 10 mois. Les capacités de mesure de ce laboratoire sont, en priorité, dédiées à la métrologie des optiques de SOLEIL. Cependant, le laboratoire est ouvert à d'autres types de caractérisation et collabore étroitement avec d'autres laboratoires de métrologie et des sociétés (12) afin 

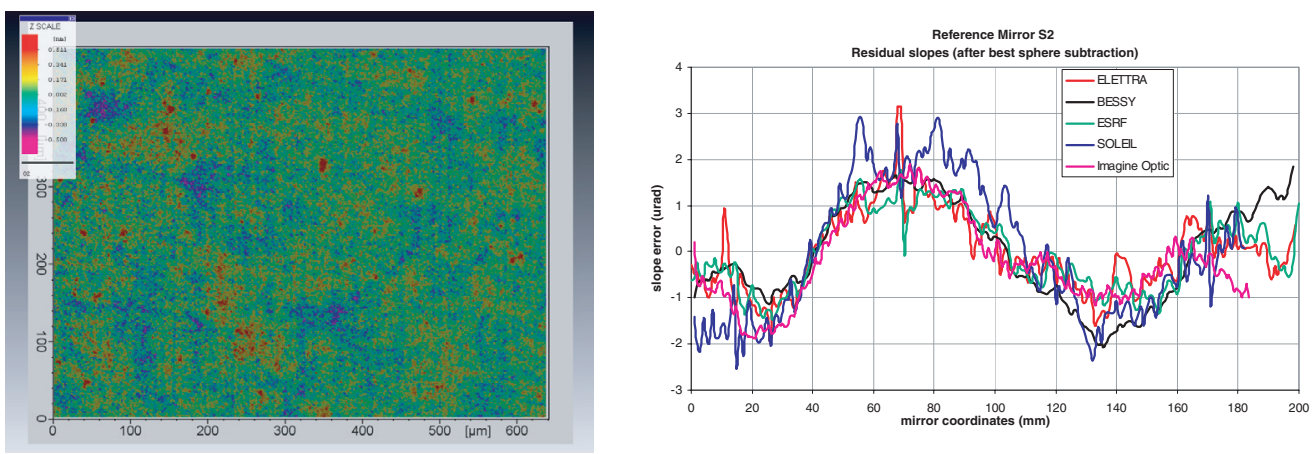

Figure 11. mesure de rugosité sur un miroir de SOLEIL (moins de $0.5 \mathrm{~nm}$ rms de rugosité) et mesure de forme comparées aux autres LTP européens [10].

de développer d'autres outils encore plus précis nécessaire à la caractérisation des futures générations d'optiques pour la nanofocalisation du rayonnement synchrotron.

Des exemples de mesures sont donnés sur la figure 11.

\section{Remerciement}

Les auteurs souhaitent remercier les membres de SOLEIL, le CEA DIF et le LNE pour leur support financier pour la construction de la ligne de METROLOGIE et TESTS. Le support continu de Oleg Chubar et du Groupe Machine de SOLEIL est également très apprécié.

\section{Références}

[1] G. Ulm, "Radiometry with synchrotron radiation". Metrologia 40 (2003) S101-S106

[2] ESRF BM05 http://www.esrf.fr/UsersAndScience/Experiments/Imaging/BM05/

[3] http://www.synchrotron-soleil.fr/anglais/science-and-users/experiments/

[4] http://www.jobinyvon.fr/frdivisions/VUV/tgm.htm

[5] Ulm G, Beckhoff B, Klein R, Krumrey M, Rabus H and Thornagel R 1998 Proc. SPIE 3444 610-21

[6] Wende B 1995/96 Metrologia 32 419-24

[7] Ulm G and Wende B 1997 R"ontgen Centennial ed A Haase,

[8] G Landwehr and E Umbach (Singapore: World Scientific) pp 81-99

[9] Schwinger J 1949 Phys. Rev. 75 1912-25

[10] A. Rommeveaux, D. Cocco, M. Thomasset, F. Siewert, First report on a European round robin for slope measuring profilers, SPIE Conference, San Diego: SPIE Proc. 5921-20, 2005, pp. 117-128

[11] M. Thomasset, S. Brochet, F. Polack, "Latest metrology results with the SOLEIL synchrotron LTP” L. Assoufid, P. Takacs, J. Taylor, eds., Proc. SPIE 5921, 12-20 (2005)

[12] Johan Floriot, Xavier Levecq, Samuel Bucourt, Muriel Thomasset, François Polack, Mourad Idir, Pascal Mercere, Thierry Moreno, X-ray mirror surface measurements with a stitching ShackHartmann profilometer, Applied Optics, to be submitted 